\section{Swyer-James syndrome in a 7-year-old female}

\author{
Jun Mori,1,2 Daisuke Kaneda,1 \\ Atsushi Fujiki,1 Kenichi Isoda,1 \\ Tomoya Kotani, 3 Yo Ushijima 3 \\ 1Department of Pediatrics, Matsushita \\ Memorial Hospital, Osaka; 2Department \\ of Pediatrics, Kyoto Prefectural \\ University of Medicine, Kyoto; \\ ${ }^{3}$ Department of Radiology, Matsushita \\ Memorial Hospital, Osaka, Japan
}

\begin{abstract}
Swyer-James syndrome is a rare syndrome that occurs as a result of repeated bronchiolitis and pneumonitis in childhood. Most cases are asymptomatic, and subsequent diagnosis may not occur until adulthood. We present the case of a 7-year-old female with Swyer-James syndrome, which was initially diagnosed and treated as asthma. The patient developed respiratory distress and atelectasis which were treated with biphasic cuirass ventilation. This case suggests that Swyer-James syndrome should be a concern in patients with chronic cough and wheezing, and highlights the importance of taking a careful history and appropriate radiological investigations for diagnosis. Once Swyer-James syndrome is diagnosed, prophylaxis and appropriate management of respiratory infections becomes important.
\end{abstract}

\section{Case Report}

The female patient, now aged seven years, was born as twin after 34 weeks gestation with a birth weight of $2.286 \mathrm{~g}$. Past medical history was significant for pneumonia twice, at 3 - and 5 - years of age. The pneumonia at 5 years of age had been thought to be due to Chlamydia pneumoniae infection, as IgM antibodies to $C$. pneumoniae were elevated. She had taken a leukotriene receptor antagonist (LTRA) for one month prior to admission because of a diagnosis of asthma by her physician. One day prior to admission, she started to cough at night and utilized inhaled budesonide, however, her symptoms did not improve. The following day, she presented to our hospital complaining of dyspnea. Body temperature was $36.9^{\circ} \mathrm{C}$, blood pressure (systolic/diastolic) was $96 / 64 \mathrm{mmHg}$, pulse was 125 beats per minute, respiratory rate was 24 breaths per minute, and oxygen saturation was $94 \%$. On physical examination, lung auscultation indicated wheezing in both inspiratory and expiratory phases, and decreased breath sounds at the left lung. The results of clinical laboratory tests were unremarkable, with the exception of elevated white blood cell count $(16,200 / \mathrm{uL})$ and slightly elevated serum levels of C-reactive protein $(0.94 \mathrm{mg} / \mathrm{dL})$. No abnormal findings were detected via posteroanterior (PA) chest radiography on admission.

The patient was initially treated for a suspected asthma attack. She was therefore administered intravenous prednisolone, inhaled $\beta_{2}$-agonist, and supplemental oxygen in addition to a LTRA. However, these treatments were not effective at resolving, or improving the presented symptoms. Consequently, the PA chest radiogram taken on admission was re-examined and decreased volume of the left hemithorax was indicated (Figure 1A). Retrospectively, the same finding was noted in the chest radiogram taken one month before the current admission. Therefore, the PA chest radiogram was reexamined on the fourth day of the admission and showed atelectasis in the middle of the left lung. Intravenous prednisolone administration was terminated. For atelectasis, biphasic cuirass ventilation (BCV) was started to promote drainage of the mucous secretions, with the use of a Respiratory Therapy External (RTX, Medivent, London, UK) device. Respiratory symptoms improved, and PA chest radiography demonstrated that atelectasis ameliorated one day after the initiation of BCV. Thorax computed tomography (CT) and CT angiography were obtained for further investigation. Hyperlucency and decreased peripheral vascular shadowing of the left lung was detected (Figure 1B,C, respectively). Tc-99m MAA lung perfusion scintigraphy was also performed. Diminished activity of the left lung was clearly indicated (Figure 1D). As a result of the radiologic findings, patient history, and clinical course, the diagnosis of Swyer-James syndrome was made. Pulmonary function testing after discharge revealed decreased V25/HT (44.1\% of predicted) with both normal forced vital capacity (FVC) (95.9\%), and the ratio of forced expiratory volume in $1 \mathrm{sec}$ (FEV1) to FVC (FEV1/FVC) (95.4\%). These results suggested mild peripheral obstructive airway disease in this patient.

\section{Discussion}

Swyer-James syndrome was first reported in 1953. ${ }^{1}$ It is a rare syndrome in which an unilateral hyperlucent lung develops due to bronchiolitis obliterans caused by viral infections such as adenovirus and/or measles.2,3 Bronchiolitis obliterans induces inflammation and fibrosis of the respiratory walls, leading to the narrow-
Correspondence: Jun Mori, Department of Pediatrics, Matsushita Memorial Hospital, 5-55 Sotojima-cho, Moriguchi-shi, Osaka, Japan.

Tel.: +81.6.6992.1231 - Fax: +81.6.6992.4845.

E-mail: jun1113@koto.kpu-m.ac.jp

Key words: Swyer-James syndrome; Biphasic cuirass ventilation; Asthma.

Contributions: JM drafted the manuscript; JM, $\mathrm{DK}, \mathrm{AF}$ and $\mathrm{KI}$ saw and managed the patient; JM, TK and YU interpreted the radiograms.

Conflict of interest: the authors declare no potential conflict of interest.

Received for publication: 15 June 2016.

Accepted for publication: 29 July 2016 .

This work is licensed under a Creative Commons Attribution NonCommercial 4.0 License (CC BYNC 4.0).

(C) Copyright J. Mori et al., 2016

Licensee PAGEPress, Italy

Pediatric Reports 2016; 8:6643

doi:10.4081/pr.2016.6643

ing of the bronchiolar lumen. Fibrosis of intraalveolar septae provokes obstruction of the pulmonary capillary bed, and decreased blood flow to the major pulmonary artery segments, resulting in a hypoplastic pulmonary artery. Consequently, the typical radiological findings are unilateral hyperlucent lung, hypoplasia of pulmonary artery and pulmonary hypoperfusion. Patients may complain of productive cough, shortness of breath and dyspnea on exertion. However, most patients are asymptomatic, thus diagnosis is usually unexpected, and confirmed on screening chest radiographies in adults. There is no radical cure for Swyer-James syndrome. In a case complicated with asthma or other respiratory pathologies, for which treatments exist, effective therapeutic management may lessen the severity of symptoms evoked by Swyer-James syndrome. As such, prophylaxis, and appropriate management of respiratory infections is important. Although the outlook of Swyer-James syndrome is excellent in many cases, a few cases require surgical management, such as pnuemonectomy, due to persistent and severe lung infection. ${ }^{4}$ An anamnestic of recurrent bacterial infections of the affected lung is an indication for surgery. ${ }^{5} \mathrm{~A}$ female patient in the present case study had productive cough and wheezing, which was likely due to the narrowing of the bronchiolar lumen caused by SwyerJames syndrome. Lung function testing showed decreased V25/HT, accompanied by normal FVC and FEV1/FVC. These data suggest V25/HT could be a sensitive marker of peripheral airway closure in the early stages of 
obstructive airway disease. At first, the patient received the diagnosis of asthma. There might be a possibility that asthma was complicated by Swyer-James syndrome. However, treatment for asthma, including intravenous administration of prednisolone and inhalation of $\beta_{2}$-agonist, were not effective. Thus, the main pathogenesis of this episode is thought to be due to airway obstruction due to SwyerJames syndrome and atelectasis. Narrowing of the bronchiolar lumen caused atelectasis, which BCV dramatically ameliorated.
Bronchoscopy is reported to be useful for removal of mucous secretions. ${ }^{6}$ However, it is an invasive procedure, and should be avoided, if possible, especially in children. BCV provides an alternative option to bronchoscopy. SwyerJames syndrome should be a concern in patients with chronic cough and wheezing, and radiological findings should carefully be examined. Once Swyer-James syndrome is diagnosed prophylaxis, and appropriate management of respiratory infections becomes important.

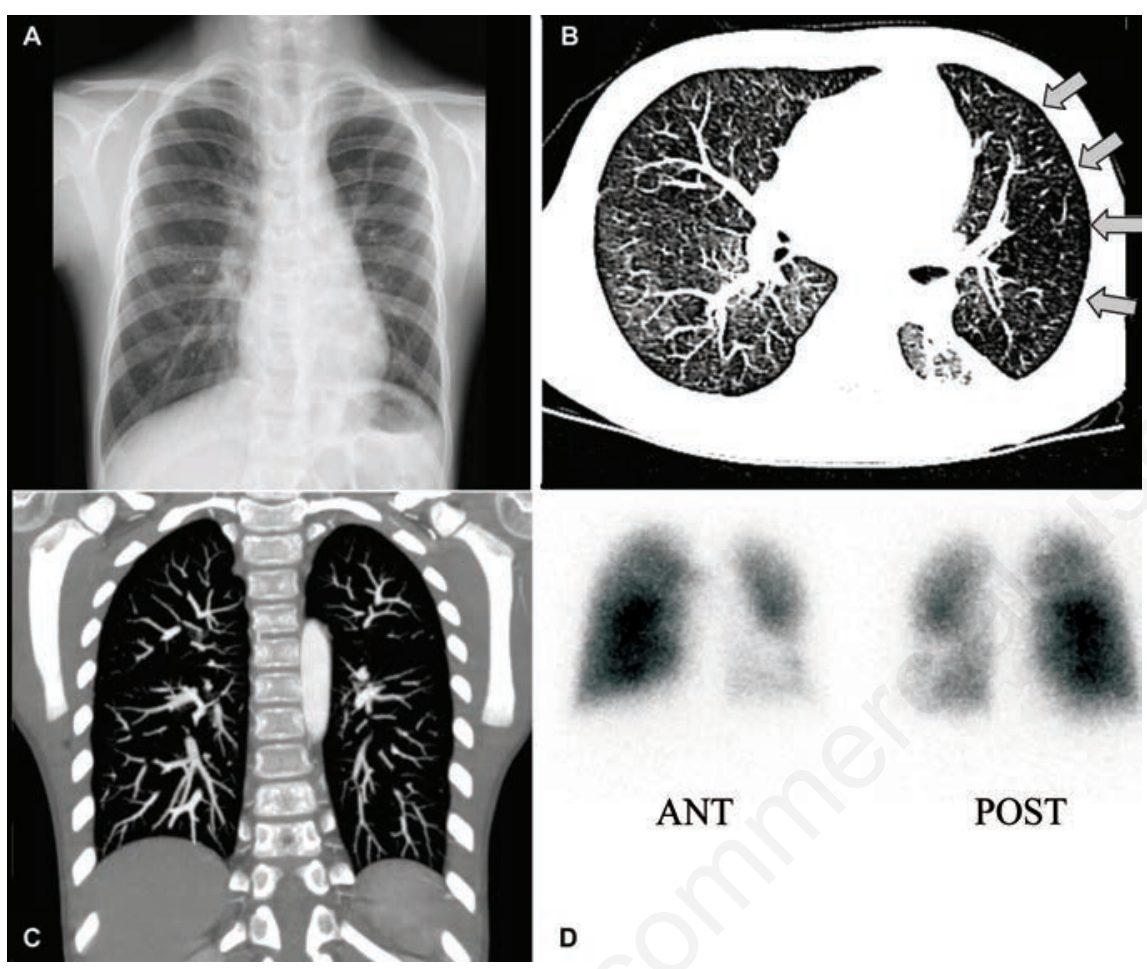

Figure 1. Radiological findings. Posterioanterior chest X-ray radiographs obtained on the admission show the decreased volume of the left hemithorax (A). Computed tomography (CT) (axial slices in lung window) demonstrates hyperlucent regions are present (B), and CT angiography indicates diminished vasculature $(\mathrm{C})$, in the left lung. Tc-99m MAA lung perfusion scanning clearly shows perfusion defects in the affected lung (D).

\section{Conclusions}

We report a case of Swyer-James syndrome in a 7-year-old female, diagnosed by chest Xray, CT angiography, lung perfusion scintigraphy and pulmonary function testing. V25/HT in pulmonary function testing could be a sensitive marker of airway closure, and BCV is an effective treatment for ateletctasis in SwyerJames syndrome. There might be some individuals with Swyer-James syndrome among patients diagnosed with asthma. Thus a careful examination of chest X-ray radiograms and pulmonary function testing is needed.

\section{References}

1. Swyer PR, James GCW. A case of unilateral pulmonary emphysema. Thorax 1953;8: 133-6.

2. Macpherson RI, Cumming GR, Chernick V. Unilateral hyperlucent lung: a complication of viral pneumonia. J Can Ass Radiol 1969;20:225-31.

3. Spigelblatt L, Rosenfeld R. Hyperlucent lung: long term complication of adenovirus type 7 pneumonia. J Can Med Ass 1983; 128:47-9.

4. Fregonese L, Girosi D, Battistini E, et al. Clinical, physiologic, and roentgenographic changes after pneumonectomy in a boy with Macleod/Swyer-James syndrome and bronchiectasis. Pediatr Pulmonol 2002; 34:412-6.

5. Vishnevsky A, Nikoladze GD. New approach to the surgical treatment of Swyer-James/ MacLeod syndrome. Ann Thorac Surg 1990; 50:103-4.

6. Preciado D, Verghese S, Choi S. Aggressive bronchoscopic management of plastic bronchitis. Int J Pediatr Otorhinolaryngol 2010; 74:820-2. 